\title{
Understanding disaster governance: An anthropological Francophone contribution
}

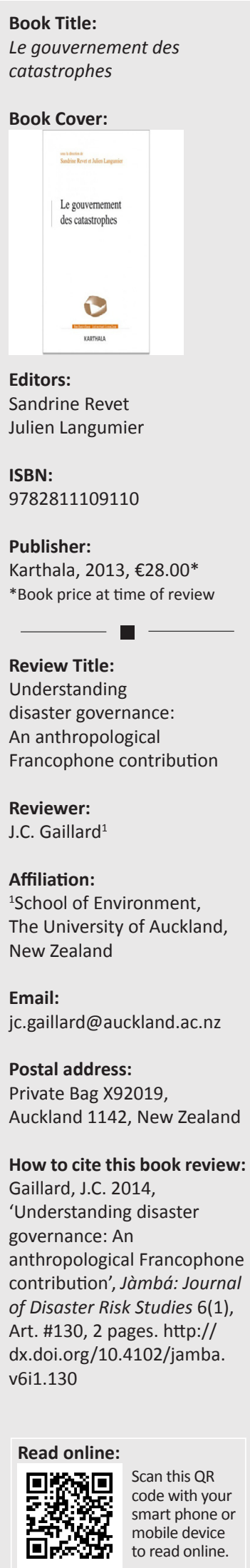

Cutting-edge books on disasters in French are seldom found. 'Le gouvernement des catastrophes' by Sandrine Revet and Julien Langumier is the exception to the rule. This edited volume indeed provides powerful reflections on how people, as well as institutional and political agencies, consider disasters, disaster risk reduction and post-disaster recovery. It grounds its approach in the traditions of anthropology in dialogue with cognate disciplines such as sociology and history.

The anthropology of disaster is a relatively new field in France and many other Francophone countries. It only emerged as an organised body of research in the 2000s under the leadership of a group of young researchers, including Sandrine Revet and Julien Langumier, the two co-editors of this book. This volume provides a synthesis of around a decade of works and reflections. It is complemented with contributions by fellow young European anthropologists who offer an interesting cross-cultural perspective. Overall, this book is a novel, welcome and pivotal addition to the Francophone literature on disasters. One that leaves the reader with more questions than answers though, for it is indeed an intellectual academic exercise which does not seek to provide any recommendations for policy or practice.

The book is a dense compilation of six case studies preceded by a framing chapter by the co-editors and followed by a post-face reflection by Nicolas Dodier. It is articulated around three sections, each of which includes two chapters. The first section focuses on 'anticipation, preparation and controversies' and contains studies on debris flows and tourism in Kazakhstan in the 1960s, by Marc Elie, as well as an ethnography of the avian flu by Frédéric Keck. The second section discusses the role and participation of local communities to disaster risk reduction and postdisaster recovery through case studies of Sri Lanka in the aftermath of the 2004 tsunami, by Mara Benadusi, and south-eastern France confronted with recurrent flooding along the Rhône River by Julien Langumier. The final section deals with the memory and commemoration of disasters and consists in chapters on repeated floods in Santa Fe, Argentina, by Susan Ullberg, and the 1976 chemical plant accident in Seveso, Italy, by Laura Centemeri. All chapters are long enough for their authors to provide both ample empirical materials and detailed theoretical reflections.

In the words of Sandrine Revet and Julien Langumier in their excellent introductory chapter, all studies examine the social and institutional mechanisms established to, (1) prevent disasters through 'controlling uncertainty and governing the future' (p. 14) and (2) prepare to minimise losses through 'surveillance and warning' (p. 14) should disasters be meant to occur. They particularly argue against two widely accepted ideas which have been guiding disaster research as well as policies and practices of disaster risk reduction and post-disaster recovery over the past few decades.

The first of these ideas is that there is a 'risk culture' or 'disaster subculture' amongst 'modern societies' and those frequently affected by disasters, which policymakers and practitioners often translate into a 'culture of prevention' required to foster disaster risk reduction. This is a prominent theme in Francophone disaster research and French disaster risk reduction policies, which is adequately challenged in this book, notably in Julien Langumier's chapter. The second idea is that there is such a thing as a 'community' to provide a local platform for disaster risk reduction through the participation of those at risk. Here, notably in Mara Benadusi's chapter, the argument is directed to the discourse and skewed practice of community-based activities, as an outcome, rather than to the fundamentals of people's participation, as a process, in disaster risk reduction.

The book also aims to ground people's and institutions' response to disaster in a long-term, everyday perspective through different ethnographic postures. The first consists in fine-grained case studies of particular events in their local contexts, as astutely demonstrated in Julien Langumier's chapter. The second stance looks at local events in the context of wider interventions by external stakeholders of disaster risk reduction and post-disaster recovery, as in Frédéric Keck's

Copyright: ( 2014. The Authors. Licensee: AOSIS OpenJournals. This work is licensed under the Creative Commons Attribution License. 
study. Finally, the third approach, fostered, for example, in Laura Centemeri's chapter, provides a long-term view of changes in the interactions between people and institutions. Noteworthy, though, is that most chapters combine these different perspectives in multidimensional ethnographies of disaster events. For example, Susan Ullberg's study relies upon a long-term fine-grained approach, whilst Mara Benadusi blends local ethnography with a multiscalar approach. It should also be noted, however, that case studies discussed in this book emphasise large-scale, 'exceptional' disasters. An exciting next step would be, henceforth, to apply the foregoing approaches to small-scale events, the cumulative impact of which is likely to be higher than that of large-scale disasters, even though they are absent from most policies geared towards reducing the risk of disaster.

It is important to acknowledge that the issues discussed in the different chapters of the book are not novel per se. They have all been discussed in Anglophone literature - some to great extents such as the skewed approach of participation in community-based disaster risk reduction and postdisaster recovery or the prominence of hazards in planning development policies. In fact, for all chapters, an overall better grounding in the relevant Anglophone literature may have allowed for an interesting dialogue and mutual enrichment.
The main contribution of this volume is, however, elsewhere. It springs from the integration of all issues in a standalone cohesive piece of scholarship, not a patchwork of miscellaneous studies hastily pulled together in an edited book. Sandrine Revet and Julien Langumier's introductory chapter, as well as Nicolas Dodier's conclusion, exemplify this integrated approach, which is also evident in the effort made by all authors to use common terminologies and shared frameworks.

The book further stands out because of its compelling ability to bridge some of the artificial divides which have hampered our understanding of disasters over the past few decades. Chapters collated in this volume refer to places from both 'North' and 'South' and discuss 'natural', 'biological' and 'technological' hazards all together, showing similarities and dissimilarities in people's and institutions' responses to disasters.

In conclusion, 'Le gouvernment des catastrophes' is a very stimulating read, which will, for sure, become a classic within Francophone literature on disasters. Scholars who do not read French should look out for the English edition to be published shortly. 